\title{
Exact and Inexact Hummel-Seebeck Type Method for Variational Inclusions
}

\author{
Steeve Burnet, Célia Jean-Alexis and Alain Piétrus \\ Laboratoire LAMIA, EA4540, Université des Antilles, Département de Mathématiques et Informatique, Campus \\ de Fouillole, 97159 Pointe-à-Pitre - France \\ Email: sburnet@martinique.univ-ag.fr, cjeanale@univ-ag.fr, apietrus@univ-ag.fr
}

\begin{abstract}
We deal with a perturbed version of a Hummel-Seebeck type method to approximate a solution of variational inclusions of the form : $0 \in \Phi(z)+F(z)$ where $\Phi$ is a single-valued function twice continuously Fréchet differentiable and $F$ is a set-valued map from $\mathbb{R}^{n}$ to the closed subsets of $\mathbb{R}^{n}$. This framework is convenient to treat in a unified way standard sequential quadratic programming, its stabilized version, sequential quadratically constrained quadratic programming, and linearly constrained Lagrangian methods (see [1]). We obtain, thanks to some semistability and another property (which is close to the hemistability) of the solution $\bar{z}$ of the previous inclusion, the local existence of a sequence that is superquadratically or cubically convergent.
\end{abstract}

Keywords: Set-valued mapping, generalized equations, semistability, superquadratic convergence, cubic convergence.

\section{Introduction}

In this paper, we study Hummel-Seebeck type and inexact Hummel-Seebeck type methods for approximating a solution of the variational inclusion

$$
0 \in \Phi(z)+F(z)
$$

where $\Phi$ is a single-valued function and $F$ is a set-valued map from $\mathbb{R}^{n}$ to the closed subsets of $\mathbb{R}^{n}$.

Variational inclusions are an abstract model of a wide variety of variational problems including linear and non-linear complementarity problems, systems of non-linear equations, variational inequalities (for example, first-order necessary conditions for nonlinear programming), etc. In particular, they may characterize optimality or equilibrium and then have several applications in engineering (analysis of elastoplastic structures, traffic equilibrium problems, etc.) and economics (Walrasian equilibrium, Nash equilibrium). For further details on such applications one can refer to [2].

In the last decade, several iterative methods to solve the inclusion (1) have been introduced. These methods consist in generating an iterative sequence $\left(z_{k}\right)$ obtained by subsequently solving implicit subproblems of the form $0 \in A\left(z_{k}, z_{k+1}\right)+F\left(z_{k+1}\right)$, where $A$ denotes some approximation of the mapping $\Phi$. When the Fréchet derivative $\Phi^{\prime}$ of the function $\Phi$ is locally Lipschitz, Dontchev [3,4] associates to (1) a Newton-type method based on a partial linearization, which is locally quadratically convergent. Following his work, Piétrus [5] obtains a Newton-type sequence which converges whenever $\Phi^{\prime}$ satisfies a Hölder-type condition and in [6] he proves the stability. Using a second-degree Taylor polynomial expansion of $\Phi$ at $z_{k}$, Geoffroy, Hilout and Piétrus [7] introduced a method involving the second order Fréchet derivative and, when $\Phi^{\prime \prime}$ is Lipschitz, they obtained a cubic convergence. In [8], they proved the stability of the method and in [9] Geoffroy and Piétrus showed that the previous method is superquadratic when $\Phi^{\prime \prime}$ satisfies a Hölder condition. Jean-Alexis presented in [10] a method without second order Fréchet derivative, which is also cubically convergent and Geoffroy, Jean-Alexis and Piétrus showed the stability of this method in [11]. Lately, Cabuzel and Piétrus [12] proposed a generalization of these methods by taking more iterates. They actually associated to (1) the relation

$$
0 \in \Phi\left(z_{k}\right)+\sum_{i=1}^{N} a_{i} \Phi^{\prime}\left(z_{k}+\beta_{i}\left(z_{k+1}-z_{k}\right)\right)\left(z_{k+1}-z_{k}\right)+F\left(z_{k+1}\right),
$$


where $N \geq 1,\left(a_{i}\right)_{1 \leq i \leq N}$ and $\left(\beta_{i}\right)_{1 \leq i \leq N}$ are sequences satisfying

$$
\sum_{i=1}^{N} a_{i}=1, \quad \sum_{i=1}^{N} a_{i} \beta_{i}=\frac{1}{2}
$$

and they proved the cubic convergence of this method inspired by a multipoint iteration formula given in [13] for approximating $\Phi$. Note that if $N=2, a_{1}=a_{2}=\frac{1}{2}, \beta_{1}=0, \beta_{2}=1,(2)$ is the Hummel-Seebeck type method introduced in [10]. Recently, an extension of this last method has been introduced in [14] by Rashid.

Let us point out that all these methods have been studied when a pseudo-Lipschitz property is satisfied for the set-valued map $(\Phi+F)^{-1}$ or one of its approximation. For more details on this property, the reader can refer to $[15,16,17,18,19,20,21,22,23,24]$. In addition, we can underline that it has been shown in $[25]$ that this property has some importance for the design of electrical circuits involving nonsmooth and non-monotone electronic devices like DIAC (DIode Alternating Currents).

In $[26,27,28,29]$, the authors study inclusion (1) by using an assumption which is directly connected to a solution: the semistability concept. This concept has been introduced by Bonnans [26] for variational inequalities. A solution $\bar{z}$ of a variational inclusion is said to be semistable if, given a small perturbation on the left-hand side, a solution $z$ of the perturbed variational inclusion that is sufficiently close to $\bar{z}$ is such that the distance of $z$ to $\bar{z}$ is of the order of the magnitude of the perturbation. Under this condition, in $[27,28,29]$ they proved superquadratic and cubic convergence for sequences defined respectively by the following methods

$$
\begin{gathered}
0 \in \Phi\left(z_{k}\right)+\Phi^{\prime}\left(z_{k}\right)\left(z_{k+1}-z_{k}\right)+M_{k}\left(z_{k+1}-z_{k}\right)^{2}+F\left(z_{k+1}\right), \\
0 \in \Phi\left(z_{k}\right)+\frac{1}{2}\left(M_{k}+M_{k+1}\right)\left(z_{k+1}-z_{k}\right)+F\left(z_{k+1}\right), \\
0 \in \Phi\left(z_{k}\right)+\sum_{i=1}^{N} a_{i} M_{k}^{i}\left(z_{k+1}-z_{k}\right)+F\left(z_{k+1}\right),
\end{gathered}
$$

where $M_{k}, M_{k+1}$ and $M_{k}^{i}$ are $q \times q$ matrices.

Let us note that the concept of semistability has also been used by Izmailov and Solodov in [1] in order to study the convergence of the following inexact Josephy-Newton method for solving generalized equations

$$
0 \in \Phi\left(z_{k}\right)+\Phi^{\prime}\left(z_{k}\right)\left(z_{k+1}-z_{k}\right)+\omega_{k}+F\left(z_{k+1}\right),
$$

where $\omega_{k}$ is a perturbation term and they obtained under some assumptions superlinear and quadratic convergence for the sequence $\left(z_{k}\right)$.

Recently, in [30] this concept has also been used to study the exact and the inexact Newton-type methods in the subanalytic context.

Following these works, we first consider an (exact) Hummel-Seebeck (noted (HS)) type method

$$
0 \in \Phi\left(z_{k}\right)+\frac{1}{2}\left(\Phi^{\prime}\left(z_{k}\right)+\Phi^{\prime}\left(z_{k+1}\right)\right)\left(z_{k+1}-z_{k}\right)+F\left(z_{k+1}\right),
$$

and then, an inexact Hummel-Seebeck (noted (iHS)) type method

$$
0 \in \Phi\left(z_{k}\right)+\frac{1}{2}\left(\Phi^{\prime}\left(z_{k}\right)+\Phi^{\prime}\left(z_{k+1}\right)\right)\left(z_{k+1}-z_{k}\right)+\omega_{k}+F\left(z_{k+1}\right)
$$

where $\omega_{k} \in \mathbb{R}^{n}$ is a perturbation term which has different forms and may play various roles. We obtain cubically and superquadratically convergent sequences using in addition the concept of semistability and another property which is close of the hemistability property (the assumption $(H 1)$ ). It is important to underline that the concept of hemistability as it has been introduced in [26] by Bonnans is very close to Newton's sequence. Thus, this concept is not adapted when we want to treat a problem with another sequence and it is the reason why in the context of Hummel-Seebeck, we have to introduce another property of the solution (assumption $(H 1)$ ), so we obtain existence and convergence for our sequence. 
Furthermore, we observe that the order of convergence of inexact Hummel-Seebeck type method is better than the one of inexact Josephy-Newton method proposed in [1].

The rest of this paper is organized as follows: in section 2, we define the concept of semistability of solutions, show the relations with another concepts and give a preliminary result. In section 3 , we study the convergence of Hummel-Seebeck method (3); in section 4, we consider the inexact Hummel-Seebeck method (4) with one of its extension and we give an example of problem where the methods presented can be applied.

Throughout this paper, the norms are denoted by $\|\cdot\|$ and a set-valued map $F$ from $\mathbb{R}^{n}$ to $\mathbb{R}^{n}$ is indicated by $F: \mathbb{R}^{n} \rightrightarrows \mathbb{R}^{n}$.

\section{Definitions and Background Material}

We recall the following definitions about rate of convergence.

Definition 1 Let $\left(z_{n}\right)$ be a sequence which converges towards $\bar{z}$ in a normed space. If $K_{p}:=\lim _{n \rightarrow+\infty} \frac{\left\|z_{n+1}-\bar{z}\right\|}{\left\|z_{n}-\bar{z}\right\|^{p}}$ exists and $K_{p}>0$, then $\left(z_{n}\right)$ is said to be convergent of order $p$ towards $\bar{z}$.

- When $p=1,\left(z_{n}\right)$ is said to be linearly convergent.

- When $p=2,\left(z_{n}\right)$ is said to be quadratically convergent.

- When $p=3,\left(z_{n}\right)$ is said to be cubically convergent.

If $K_{1}=0$, then $\left(z_{n}\right)$ is said to be superlinearly convergent and if $K_{2}=0$, then $\left(z_{n}\right)$ is said to be superquadratically convergent.

Let us define the notions of semistability on which this study relies on.

Definition $2 A$ solution $\bar{z}$ of (1) is said to be semistable if $c_{1}>0$ and $c_{2}>0$ exist such that, for all $(z, \delta) \in \mathbb{R}^{n} \times \mathbb{R}^{n}$, solution of

$$
\delta \in \Phi(z)+F(z)
$$

with $\|z-\bar{z}\| \leq c_{1}$, then one has $\|z-\bar{z}\| \leq c_{2}\|\delta\|$.

The following proposition gives the rate of convergence of the Hummel-Seebeck type method in the case of semistable solutions. For the proof, the reader could refer to [28].

Proposition 1 Let $\Phi: \mathbb{R}^{n} \rightarrow \mathbb{R}^{n}$ be twice continuously Fréchet differentiable and $\bar{z}$ be a semistable solution of $(1)$. Let $\left(z_{k}\right)$ be a sequence computed by (3) which converges toward $\bar{z}$. If $\Phi^{\prime \prime}$ is locally Lipschitz then $\left(z_{k}\right)$ converges cubically.

It is obvious that semistability doesn't assure the existence of sequences $\left(z_{k}\right)$ but gives only the order of convergence of sequences $\left(z_{k}\right)$ and we can remark that the semistability for $(1)$ is in fact equivalent to the strong metric subregularity for $\Phi+F$ (see [20]). But for simplicity, we prefer use the terminology of semistability in the rest of the paper. Let us also add that the semistability property is evidently implied by Robinson's strong regularity of the solution $\bar{z}$, see [23]. For more details on this concept and another one which is the hemistability, the reader can refer to [26].

\section{Convergence Analysis for the HS Method}

In this section, we consider the (exact) Hummel-Seebeck type method given by (3).

In [28], the authors proved the convergence of this sequence in assuming its existence. The next theorem gives the existence and the rate of convergence of the Hummel-Seebeck type method when we add another property.

Let us underline that existence of sequences has also been proved using the stability property in some recent papers in the context of Newton's method, proximal methods and methods using the Tikhonov regularizations. For more details on these works, the reader could refer to [31,32,33,34]. 
Theorem 1 Let $\Phi: \mathbb{R}^{n} \rightarrow \mathbb{R}^{n}$ be twice Fréchet differentiable near a point $\bar{z} \in \mathbb{R}^{n}$ such that the second order derivative $\Phi^{\prime \prime}$ is continuous at $\bar{z}$. Let $\bar{z}$ be a semistable solution of (1) and consider the following assumption:

(H1) For each $z \in \mathbb{R}^{n}$ close enough to $\bar{z}$, the inclusion

$$
0 \in \Phi(z)+\frac{1}{2}\left(\Phi^{\prime}(z)+\Phi^{\prime}(z+\zeta)\right) \zeta+F(z+\zeta)
$$

has a solution $\zeta(z)$ such that $\zeta(z) \rightarrow 0$ as $z \rightarrow \bar{z}$.

There exists $\varepsilon>0$ such that if $\left\|z_{0}-\bar{z}\right\| \leq \varepsilon$, then :

(i) At each step $k$, a solution $z_{k+1}$ of (3) satisfying $\left\|z_{k+1}-z_{k}\right\| \leq \frac{3}{2} \varepsilon$ exists.

(ii) If $\Phi^{\prime \prime}$ is locally Lipschitz, the sequence $\left(z_{k}\right)$ defined in this way converges cubically toward $\bar{z}$.

Proof: We just have to prove $(i)$ and the convergence of $\left(z_{k}\right)$ toward $\bar{z}$, then $(i i)$ will follow from Proposition 1.Assume $\Phi^{\prime \prime}$ is continuous at $\bar{z}$. Take $\varepsilon_{0} \leq \min \left(c_{1}, \frac{1}{9 c_{2}}\right)$, where $c_{1}, c_{2}$ are given by the semistability. Note that in the definition of semistability, once we have found a positive number $c_{1}$, the property stands for any positive number $c \leq c_{1}$. That is to say, without loss of generality, we can take $c_{1}$ as small as required.From the assumption $(H 1)$, we have that, for some $\varepsilon \in\left[0, c_{1}\right],\left\|z_{k}-\bar{z}\right\| \leq \varepsilon$ implies the existence of $z_{k+1}$ such that $\left\|z_{k+1}-\bar{z}\right\| \leq \varepsilon_{0}$ and

$$
0 \in \Phi\left(z_{k}\right)+\frac{1}{2}\left(\Phi^{\prime}\left(z_{k}\right)+\Phi^{\prime}\left(z_{k+1}\right)\right)\left(z_{k+1}-z_{k}\right)+F\left(z_{k+1}\right) .
$$

We write $\delta_{k} \in \Phi\left(z_{k+1}\right)+F\left(z_{k+1}\right)$, where

$$
\delta_{k}:=\Phi\left(z_{k+1}\right)-\Phi\left(z_{k}\right)-\frac{1}{2}\left(\Phi^{\prime}\left(z_{k}\right)+\Phi^{\prime}\left(z_{k+1}\right)\right)\left(z_{k+1}-z_{k}\right) .
$$

One has:

$$
\begin{aligned}
\left\|\delta_{k}\right\|= & \| \Phi\left(z_{k+1}\right)-\Phi\left(z_{k}\right)-\Phi^{\prime}\left(z_{k}\right)\left(z_{k+1}-z_{k}\right)-\frac{1}{2} \Phi^{\prime \prime}\left(z_{k}\right)\left(z_{k+1}-z_{k}\right)^{2} \\
& -\frac{1}{2}\left(\Phi^{\prime}\left(z_{k+1}\right)-\Phi^{\prime}\left(z_{k}\right)-\Phi^{\prime \prime}\left(z_{k}\right)\left(z_{k+1}-z_{k}\right)\right)\left(z_{k+1}-z_{k}\right) \| \\
\leq & \left\|z_{k+1}-z_{k}\right\|^{2} \int_{0}^{1}(1-t)\left\|\Phi^{\prime \prime}\left(z_{k}+t\left(z_{k+1}-z_{k}\right)\right)-\Phi^{\prime \prime}\left(z_{k}\right)\right\| \mathrm{d} t \\
& +\frac{1}{2}\left\|z_{k+1}-z_{k}\right\|^{2} \int_{0}^{1}\left\|\Phi^{\prime \prime}\left(z_{k}+t\left(z_{k+1}-z_{k}\right)\right)-\Phi^{\prime \prime}\left(z_{k}\right)\right\| \mathrm{d} t \\
\leq & \left\|z_{k+1}-z_{k}\right\|^{2} \int_{0}^{1}\left\|\Phi^{\prime \prime}\left(z_{k}+t\left(z_{k+1}-z_{k}\right)\right)-\Phi^{\prime \prime}\left(z_{k}\right)\right\| \mathrm{d} t \\
& +\frac{1}{2}\left\|z_{k+1}-z_{k}\right\|^{2} \int_{0}^{1}\left\|\Phi^{\prime \prime}\left(z_{k}+t\left(z_{k+1}-z_{k}\right)\right)-\Phi^{\prime \prime}\left(z_{k}\right)\right\| \mathrm{d} t .
\end{aligned}
$$

Since $\Phi^{\prime \prime}$ is continuous at $\bar{z}$, for $c_{1}$ small enough, one has

$$
\left\|\Phi^{\prime \prime}\left(z_{k}+t\left(z_{k+1}-z_{k}\right)\right)-\Phi^{\prime \prime}\left(z_{k}\right)\right\| \leq \frac{2}{9 c_{2}}
$$

then, for $c_{1}$ small enough,

$$
\left\|\delta_{k}\right\| \leq \frac{1}{3 c_{2}}\left\|z_{k+1}-z_{k}\right\|^{2} \leq \frac{1}{3 c_{2}}\left\|z_{k+1}-z_{k}\right\| .
$$

Since $\varepsilon_{0} \leq c_{1}$, the semistability condition gives

$$
\left\|z_{k+1}-\bar{z}\right\| \leq \frac{1}{3}\left\|z_{k+1}-z_{k}\right\| \leq \frac{1}{3}\left\|z_{k+1}-\bar{z}\right\|+\frac{1}{3}\left\|z_{k}-\bar{z}\right\|,
$$


hence

$$
\left\|z_{k+1}-\bar{z}\right\| \leq \frac{1}{2}\left\|z_{k}-\bar{z}\right\|
$$

and

$$
\left\|z_{k+1}-z_{k}\right\| \leq\left\|z_{k+1}-\bar{z}\right\|+\left\|z_{k}-\bar{z}\right\| \leq \frac{3}{2} \varepsilon
$$

These prove $(i)$ (which implies the existence of the sequence $\left(z_{k}\right)$ ) and the convergence of the sequence $\left(z_{k}\right)$.

\section{Convergence Analysis for the iHS Method}

We now consider the inexact Hummel-Seebeck type method given by (4).

Our study will be done in two parts. First of all, we will suppose the convergence of the sequence $\left(z_{k}\right)$ and we give some interesting results on the order of convergence under some property of semistability of the solution and some estimations on the perturbation terms. Then, we will present our convergence result.

Proposition 2 Let a mapping $\Phi: \mathbb{R}^{n} \rightarrow \mathbb{R}^{n}$ be twice Fréchet differentiable in a neighborhood of $\bar{z} \in \mathbb{R}^{n}$, with its second order derivative $\Phi^{\prime \prime}$ being continuous at $\bar{z}$ and let $\bar{z}$ be a semistable solution of (1). Let a sequence $\left(z_{k}\right) \subset \mathbb{R}^{n}$ be convergent to $\bar{z}$. Assume that $z_{k+1}$ satisfies (4) for all $k \in \mathbb{N}$, with some $\omega_{k} \in \mathbb{R}^{n}$ such that

$$
\left\|\omega_{k}\right\|=o\left(\left\|z_{k+1}-z_{k}\right\|^{2}+\left\|z_{k+1}-z_{k}\right\|\left\|z_{k}-\bar{z}\right\|+\left\|z_{k}-\bar{z}\right\|^{2}\right) .
$$

Then the rate of convergence of $\left(z_{k}\right)$ is superquadratic. Moreover, the rate of convergence is cubic if $\Phi^{\prime \prime}$ is locally L-Lipschitz-continuous with respect to $\bar{z}$, and provided

$$
\left\|\omega_{k}\right\|=O\left(\left\|z_{k+1}-z_{k}\right\|^{3}+\left\|z_{k+1}-z_{k}\right\|^{2}\left\|z_{k}-\bar{z}\right\|+\left\|z_{k+1}-z_{k}\right\|\left\|z_{k}-\bar{z}\right\|^{2}+\left\|z_{k}-\bar{z}\right\|^{3}\right) .
$$

Proof: If $z_{k}=\bar{z}$ for some $k$, semistability of $\bar{z}$ implies that $z_{k}=\bar{z}$ for all subsequent values of $k$ and the assertions hold trivially. Therefore, we assume that $z_{k} \neq \bar{z}$ for all $k \in \mathbb{N}$. For all $k$, one has

$$
r_{k} \in \Phi\left(z_{k+1}\right)+F\left(z_{k+1}\right),
$$

with

$$
r_{k}=\Phi\left(z_{k+1}\right)-\Phi\left(z_{k}\right)-\frac{1}{2}\left(\Phi^{\prime}\left(z_{k}\right)+\Phi^{\prime}\left(z_{k+1}\right)\right)\left(z_{k+1}-z_{k}\right)-\omega_{k}
$$

Then we get

$$
\begin{aligned}
\left\|r_{k}\right\|= & \| \Phi\left(z_{k+1}\right)-\Phi\left(z_{k}\right)-\Phi^{\prime}\left(z_{k}\right)\left(z_{k+1}-z_{k}\right)-\frac{1}{2} \Phi^{\prime \prime}\left(z_{k}\right)\left(z_{k+1}-z_{k}\right)^{2} \\
& -\frac{1}{2}\left(\Phi^{\prime}\left(z_{k+1}\right)-\Phi^{\prime}\left(z_{k}\right)-\Phi^{\prime \prime}\left(z_{k}\right)\left(z_{k+1}-z_{k}\right)\right)\left(z_{k+1}-z_{k}\right)-\omega_{k} \| \\
\leq & \left\|z_{k+1}-z_{k}\right\|^{2} \int_{0}^{1}(1-t)\left\|\Phi^{\prime \prime}\left(z_{k}+t\left(z_{k+1}-z_{k}\right)\right)-\Phi^{\prime \prime}\left(z_{k}\right)\right\| \mathrm{d} t \\
& +\frac{1}{2}\left\|z_{k+1}-z_{k}\right\|^{2} \int_{0}^{1}\left\|\Phi^{\prime \prime}\left(z_{k}+t\left(z_{k+1}-z_{k}\right)\right)-\Phi^{\prime \prime}\left(z_{k}\right)\right\| \mathrm{d} t+\left\|\omega_{k}\right\| \\
\leq & \frac{3}{2}\left\|z_{k+1}-z_{k}\right\|^{2} \int_{0}^{1}\left\|\Phi^{\prime \prime}\left(z_{k}+t\left(z_{k+1}-z_{k}\right)\right)-\Phi^{\prime \prime}\left(z_{k}\right)\right\| \mathrm{d} t+\left\|\omega_{k}\right\| \\
\leq & \frac{3}{2}\left\|z_{k+1}-z_{k}\right\|^{2} \sup _{t \in[0,1]}\left\{\left\|\Phi^{\prime \prime}\left(z_{k}+t\left(z_{k+1}-z_{k}\right)\right)-\Phi^{\prime \prime}\left(z_{k}\right)\right\|\right\}+\left\|\omega_{k}\right\| \\
= & o\left(\left\|z_{k+1}-z_{k}\right\|^{2}+\left\|z_{k+1}-z_{k}\right\|\left\|z_{k}-\bar{z}\right\|+\left\|z_{k}-\bar{z}\right\|^{2}\right) .
\end{aligned}
$$


Then, by semistability of $\bar{z}$, it holds that

$$
\begin{aligned}
\left\|z_{k+1}-\bar{z}\right\| & =O\left(\left\|r_{k}\right\|\right) \\
& =o\left(\left\|z_{k+1}-\bar{z}\right\|^{2}+\left\|z_{k+1}-\bar{z}\right\|\left\|z_{k}-\bar{z}\right\|+\left\|z_{k}-\bar{z}\right\|^{2}\right),
\end{aligned}
$$

i.e.

$$
\begin{aligned}
0 & =\lim _{k \rightarrow \infty} \frac{\left\|z_{k+1}-\bar{z}\right\|}{\left\|z_{k+1}-\bar{z}\right\|^{2}+\left\|z_{k+1}-\bar{z}\right\|\left\|z_{k}-\bar{z}\right\|+\left\|z_{k}-\bar{z}\right\|^{2}} \\
& =\lim _{k \rightarrow \infty} \frac{1}{\left\|z_{k+1}-\bar{z}\right\|+\left\|z_{k}-\bar{z}\right\|+\frac{\left\|z_{k}-\bar{z}\right\|^{2}}{\left\|z_{k+1}-\bar{z}\right\|}} .
\end{aligned}
$$

The latter relation implies that

$$
\lim _{k \rightarrow \infty} \frac{\left\|z_{k}-\bar{z}\right\|^{2}}{\left\|z_{k+1}-\bar{z}\right\|}=\infty
$$

i.e.

$$
\left\|z_{k+1}-\bar{z}\right\|=o\left(\left\|z_{k}-\bar{z}\right\|^{2}\right),
$$

which shows the superquadratic convergence rate of $\left(z_{k}\right)$.

Furthermore, if $\Phi^{\prime \prime}$ is locally $L$-Lipschitz-continuous with respect to $\bar{z}$, we get

$$
\begin{aligned}
\left\|r_{k}\right\| & \leq \frac{3}{2}\left\|z_{k+1}-z_{k}\right\|^{2} \int_{0}^{1}\left\|\Phi^{\prime \prime}\left(z_{k}+t\left(z_{k+1}-z_{k}\right)\right)-\Phi^{\prime \prime}\left(z_{k}\right)\right\| \mathrm{d} t+\left\|\omega_{k}\right\| \\
\leq & \frac{3}{4} L\left\|z_{k+1}-z_{k}\right\|^{3}+\left\|\omega_{k}\right\| \\
= & O\left(\left\|z_{k+1}-z_{k}\right\|^{3}+\left\|z_{k+1}-z_{k}\right\|^{2}\left\|z_{k}-\bar{z}\right\|+\left\|z_{k+1}-z_{k}\right\|\left\|z_{k}-\bar{z}\right\|^{2}\right. \\
& \left.+\left\|z_{k}-\bar{z}\right\|^{3}\right) .
\end{aligned}
$$

Then by semistability of $\bar{z}$, we obtain

$$
\begin{aligned}
\left\|z_{k+1}-\bar{z}\right\| & =O\left(\left\|r_{k}\right\|\right) \\
& =O\left(\left\|z_{k+1}-\bar{z}\right\|^{3}+\left\|z_{k+1}-\bar{z}\right\|^{2}\left\|z_{k}-\bar{z}\right\|+\left\|z_{k+1}-\bar{z}\right\|\left\|z_{k}-\bar{z}\right\|^{2}\right. \\
& \left.+\left\|z_{k}-\bar{z}\right\|^{3}\right)
\end{aligned}
$$

which means that the quantities

$$
\begin{gathered}
\frac{\left\|z_{k+1}-\bar{z}\right\|}{\left\|z_{k+1}-\bar{z}\right\|^{3}+\left\|z_{k+1}-\bar{z}\right\|^{2}\left\|z_{k}-\bar{z}\right\|+\left\|z_{k+1}-\bar{z}\right\|\left\|z_{k}-\bar{z}\right\|^{2}+\left\|z_{k}-\bar{z}\right\|^{3}} \\
=\frac{1}{\left\|z_{k+1}-\bar{z}\right\|^{2}+\left\|z_{k+1}-\bar{z}\right\|\left\|z_{k}-\bar{z}\right\|+\left\|z_{k}-\bar{z}\right\|^{2}+\frac{\left\|z_{k}-\bar{z}\right\|^{3}}{\left\|z_{k+1}-\bar{z}\right\|}}
\end{gathered}
$$

form a bounded sequence. But the latter is possible only when there exists $\gamma>0$ such that

$$
\frac{\left\|z_{k}-\bar{z}\right\|^{3}}{\left\|z_{k+1}-\bar{z}\right\|} \geq \gamma \text { for all } k \in \mathbb{N},
$$

i.e.

$$
\left\|z_{k+1}-\bar{z}\right\| \leq \frac{1}{\gamma}\left\|z_{k}-\bar{z}\right\|^{3} \text { for all } k \in \mathbb{N}
$$

which gives the cubic convergence rate of $\left(z_{k}\right)$.

Now, we study iteration subproblem of the form

$$
0 \in \Phi\left(z_{k}\right)+\frac{1}{2}\left(\Phi^{\prime}\left(z_{k}\right)+\Phi^{\prime}(z)\right)\left(z-z_{k}\right)+\Omega\left(z_{k}, z-z_{k}\right)+F(z),
$$


where $\Omega: \mathbb{R}^{n} \times \mathbb{R}^{n} \rightrightarrows \mathbb{R}^{n}$ is a given multifunction. In other words, the perturbation term appearing in (4) must satisfy the inclusion $\omega_{k} \in \Omega\left(z_{k}, z_{k+1}-z_{k}\right)$. As we saw in Proposition 2, semistability gives us an interesting rate of convergence assuming that the sequences $\left(z_{k}\right)$ and $\left(w_{k}\right)$ are given but it seems not to be sufficient to guarantee the existence of a sequence which converges toward a solution $\bar{z}$ of (1). However, considering some property ((H2) which is close to the hemistability property) in addition to semistability of $\bar{z}$, we obtain the following result which is the main result of this part.

Theorem 2 Let $\Phi: \mathbb{R}^{n} \rightarrow \mathbb{R}^{n}$ be twice Fréchet differentiable near a point $\bar{z} \in \mathbb{R}^{n}$, and suppose that its second order derivative $\Phi^{\prime \prime}$ is continuous at $\bar{z}$. Assume that $\bar{z}$ is a semistable solution of (1). Let $\Omega: \mathbb{R}^{n} \times \mathbb{R}^{n} \rightrightarrows \mathbb{R}^{n}$ be a multifunction satisfying the following assumptions:

(H2) For each $z \in \mathbb{R}^{n}$ close enough to $\bar{z}$, the inclusion

$$
0 \in \Phi(z)+\frac{1}{2}\left(\Phi^{\prime}(z)+\Phi^{\prime}(z+\zeta)\right) \zeta+\Omega(z, \zeta)+F(z+\zeta)
$$

has a solution $\zeta(z)$ such that $\zeta(z) \rightarrow 0$ as $z \rightarrow \bar{z}$.

(H3) The estimate

$$
\|\omega\|=o\left(\|\zeta\|^{2}+\|\zeta\|\|z-\bar{z}\|+\|z-\bar{z}\|^{2}\right)
$$

holds uniformly for $\omega \in \Omega(z, \zeta), z \in \mathbb{R}^{n}$ and $\zeta \in \mathbb{R}^{n}$ close enough to zero and satisfying

$$
0 \in \Phi(z)+\frac{1}{2}\left(\Phi^{\prime}(z)+\Phi^{\prime}(z+\zeta)\right) \zeta+\omega+F(z+\zeta) .
$$

Then there exists $\delta>0$ such that for any starting point $z_{0} \in \mathbb{R}^{n}$ close enough to $\bar{z}$, there exists a trajectory $\left(z_{k}\right) \subset \mathbb{R}^{n}$ such that $z_{k+1}$ is a solution of $(10)$ for all $k \in \mathbb{N}$ satisfying

$$
\left\|z_{k+1}-z_{k}\right\| \leq \delta
$$

any such trajectory converges to $\bar{z}$ and the rate of convergence is superquadratic. Moreover, the rate of convergence is cubic if $\Phi^{\prime \prime}$ is locally Lipschitz-continuous at $\bar{z}$, and provided (12) can be replaced by the estimate

$$
\|\omega\|=O\left(\|\zeta\|^{3}+\|\zeta\|^{2}\|z-\bar{z}\|+\|\zeta\|\|z-\bar{z}\|^{2}+\|z-\bar{z}\|^{3}\right) .
$$

Proof: For the proof, we follow the different steps of the one given in [1]. Semistability of $\bar{z}$ implies the existence of $\delta_{1}>0$ and $M>0$ such that for any $r \in \mathbb{R}^{n}$ and any $z(r)$ such that

$$
r \in \Phi(z(r))+F(z(r))
$$

and satisfying $\|z(r)-\bar{z}\| \leq \delta_{1}$, it holds that

$$
\|z(r)-\bar{z}\| \leq M\|r\|
$$

Fix any $\left.\left.\delta_{2} \in\right] 0, \delta_{1}\right]$. According to assumption $(H 2)$, there exists $\left.\left.\delta \in\right] 0, \frac{3 \delta_{2}}{5}\right]$ such that $\left\|z_{k}-\bar{z}\right\| \leq \frac{2 \delta}{3} \Rightarrow$ $\left\|\zeta\left(z_{k}\right)\right\| \leq \frac{3 \delta_{2}}{5}$. Putting $z_{k+1}=z_{k}+\zeta\left(z_{k}\right)$, we obtain the existence of a solution $z_{k+1}$ of (10) such that $\left\|z_{k+1}-\bar{z}\right\| \leq \delta_{2}$. Then one has

$$
r_{k} \in \Phi\left(z_{k+1}\right)+F\left(z_{k+1}\right),
$$

with $r_{k}$ defined in (8) with some $\omega_{k} \in \Omega\left(z_{k}, z_{k+1}-z_{k}\right)$. The inequality (9) and condition (12) imply that

$$
\left\|r_{k}\right\|=o\left(\left\|z_{k+1}-z_{k}\right\|^{2}+\left\|z_{k+1}-z_{k}\right\|\left\|z_{k}-\bar{z}\right\|+\left\|z_{k}-\bar{z}\right\|^{2}\right),
$$

then for sufficiently small value of $\delta_{2}$ (and also of $\delta$ ), we have

$$
\begin{aligned}
\left\|r_{k}\right\| & \leq \frac{2}{15 M}\left(\left\|z_{k+1}-z_{k}\right\|^{2}+\left\|z_{k+1}-z_{k}\right\|\left\|z_{k}-\bar{z}\right\|+\left\|z_{k}-\bar{z}\right\|^{2}\right) \\
\left\|r_{k}\right\| & \leq \frac{1}{5 M}\left(\left\|z_{k+1}-z_{k}\right\|^{2}+\left\|z_{k}-\bar{z}\right\|^{2}\right) \\
& \leq \frac{1}{5 M}\left(\left\|z_{k+1}-z_{k}\right\|+\left\|z_{k}-\bar{z}\right\|\right) .
\end{aligned}
$$


Since $\delta_{2} \leq \delta_{1}$, (16) holds with $r=r_{k}$ for $z_{k+1}=z\left(r_{k}\right)$. Hence, taking into account (17), we obtain

$$
\begin{aligned}
\left\|z_{k+1}-\bar{z}\right\| & \leq \frac{1}{5}\left\|z_{k+1}-z_{k}\right\|+\frac{1}{5}\left\|z_{k}-\bar{z}\right\| \\
& \leq \frac{1}{5}\left\|z_{k+1}-\bar{z}\right\|+\frac{2}{5}\left\|z_{k}-\bar{z}\right\|
\end{aligned}
$$

or equivalently

$$
\left\|z_{k+1}-\bar{z}\right\| \leq \frac{1}{2}\left\|z_{k}-\bar{z}\right\|
$$

which, in turn, implies that

$$
\left\|z_{k+1}-\bar{z}\right\| \leq \frac{1}{3} \delta
$$

Hence,

$$
\begin{aligned}
\left\|z_{k+1}-z_{k}\right\| & \leq\left\|z_{k+1}-\bar{z}\right\|+\left\|z_{k}-\bar{z}\right\| \\
& \leq \delta
\end{aligned}
$$

We thus prove that if $\left\|z_{k}-\bar{z}\right\| \leq \frac{2 \delta}{3}$ then (10) has a solution $z_{k+1}$ satisfying (14). Suppose now that $\left\|z_{k}-\bar{z}\right\| \leq \frac{2 \delta}{3}$, and $z_{k+1}$ is any solution of (10) satisfying (14) then

$$
\begin{aligned}
\left\|z_{k+1}-\bar{z}\right\| & \leq\left\|z_{k+1}-z_{k}\right\|+\left\|z_{k}-\bar{z}\right\| \\
& \leq \frac{5}{3} \delta \\
& \leq \delta_{2} \leq \delta_{1} .
\end{aligned}
$$

Thus, $z_{k+1}=z\left(r_{k}\right)$ satisfies (16) with $r=r_{k}$, and by the same argument as above, the latter implies (18) and (19). Therefore, if $\left\|z_{0}-\bar{z}\right\| \leq \frac{2 \delta}{3}$ then the next iterate $z_{1}$ can be chosen in such a way that (14) would hold with $k=0$ and any such choice will give (18) and (19) with $k=0$. The latter implies that $\left\|z_{1}-\bar{z}\right\| \leq \frac{2 \delta}{3}$. Hence, the next iterate $z_{2}$ can be chosen in such a way that (14) would hold with $k=1$ and any such choice will give (18) and (19) with $k=1$. Continuing this argument, we obtain that there exists a trajectory $\left(z_{k}\right)$ such that for each $k, z_{k+1}$ is a solution of (10) satisfying (14) and for any such trajectory (18) is valid for all $k$. But the latter implies that $\left(z_{k}\right)$ converges to $\bar{z}$. To complete the proof (with respect to the rate of convergence), it suffices to use Proposition 2.

One can note that for exact method (i.e., when $\Omega(\cdot)=\{0\}$ ), Theorem 2 reduces to Theorem 1.

It is worth noting that the concept of semistability and hemistability occurs in many nonlinear programming problems. For instance, consider the standard following mathematical programming problem coming from $[1]$ :

$$
\text { Minimize } \quad h(x) \quad \text { subject to } f(x)=0, \quad g(x) \leq 0,
$$

where $h: \mathbb{R}^{n} \rightarrow \mathbb{R}$ is a smooth function, $f: \mathbb{R}^{n} \rightarrow \mathbb{R}^{l}$ and $g: \mathbb{R}^{n} \rightarrow \mathbb{R}^{m}$ are smooth mappings. Stationary points of problem (20) and the associated Lagrange multipliers are characterized by the Karush-Kuhn-Tucker (KKT) optimality system

$$
\frac{\partial L}{\partial x}(x, \lambda, \mu)=0, f(x)=0, \mu \geq 0, g(x) \leq 0,\langle\mu, g(x)\rangle=0,
$$

where $L: \mathbb{R}^{n} \times \mathbb{R}^{l} \times \mathbb{R}^{m} \rightarrow \mathbb{R}$ defined by $L(x, \lambda, \mu)=h(x)+\langle\lambda, f(x)\rangle+\langle\mu, g(x)\rangle$ is the Lagrangian function of problem (20). More details on these notions are developed in [35]. KKT system (21) can be written in the form (1) with $z=(x, \lambda, \mu) \in \mathbb{R}^{s}=\mathbb{R}^{n} \times \mathbb{R}^{l} \times \mathbb{R}^{m}$,

$$
\Phi(z)=\left(\frac{\partial L}{\partial x}(x, \lambda, \mu), f(x), g(x)\right)
$$


and

$$
F(z)=\left\{\{0\} \times\{0\} \times\left\{y \in \mathbb{R}_{+}^{m} \mid\langle\mu, y\rangle=0\right\}, \quad \text { if } \mu \geq 0 ; \quad \emptyset \quad\right. \text { otherwise. }
$$

Then, for a given $z_{k}=\left(x_{k}, \lambda_{k}, \mu_{k}\right) \in \mathbb{R}^{s}$, the KKT system can be studied using the methods presented in this paper. Let $\bar{z} \in \mathbb{R}^{n}$ be a stationary point of problem $(20)$ and let $(\bar{\lambda}, \bar{\mu}) \in \mathbb{R}^{n} \times \mathbb{R}^{m}$ such that $(\bar{x}, \bar{\lambda}, \bar{\mu})$ satisfies (21). In assuming that $h, f$ and $g$ are three times Fréchet differentiable at $\bar{x}$, the MangarasarianFromovitz constraint qualification at $\bar{x}$ consists of saying that rank $f^{\prime}(\bar{x})=l$ and there exists $\bar{\xi} \in \operatorname{ker} g^{\prime}(\bar{x})$ such that $g_{A(\bar{x})}^{\prime}(\bar{x}) \bar{\xi}<0$, where $A(\bar{x})=\left\{i=1, \ldots, m \mid g_{i}(\bar{x})=0\right\}$ is the set of constraints active at $\bar{x}$.

In $[26,1]$, the following properties have been established: - if the solution $\bar{z}$ of (1) is semistable then $\bar{x}$ satisfies the strict Mangasarian-Fromovitz constraint qualification for $(\bar{\lambda}, \bar{\mu})$, - if the strict MangasarianFromovitz constraint qualifies at $\bar{x}$ for $(\bar{\lambda}, \bar{\mu})$ and if the second order sufficient optimality condition holds then $\bar{z}$ is semistable, - if $\bar{x}$ is a local solution of problem (20) then the second order sufficient optimality condition is also necessary for semistability of $\bar{z}$. In this case, the second order sufficient optimality condition is:

$$
\frac{\partial L}{\partial x}(\bar{x}, \bar{\lambda}, \bar{\mu})[\xi, \xi]>0 \quad \forall \xi \in C(\bar{x}) \backslash\{0\}
$$

where

$$
C(\bar{x})=\left\{\xi \in \mathbb{R}^{n} \mid f^{\prime}(\bar{x}) \xi=0, g_{A(\bar{x})}^{\prime}(\bar{x}) \xi \leq 0,\left\langle f^{\prime}(\bar{x}), \xi\right\rangle \leq 0\right\}
$$

is the critical cone of the problem $(20)$ at $\bar{x}$.

Thus, it follows from Theorems 1 and 2 that cubic and superquadratic convergence of methods for problem (20) are guaranteed under the strict Mangasarian-Fromovitz constraint qualification and the second order sufficient optimality condition. For more details, the reader can refer to [1].

Acknowledgments. We would like to thank the anonymous referee for his valuable suggestions and remarks that enabled us to improve the presentation of this manuscript.

\section{References}

1. A. Izmailov and M. Solodov, "Inexact josephy-newton framework for generalized equations and its applications to local analysis of newtonian methods for constrained optimization," Comput. Optim. Appl., vol. 46, no. 2, pp. 347-368, 2010.

2. M. Ferris and J. Pang, "Engineering and economic applications of complementarity problems," SIAM Rev., vol. 39, no. 4, pp. 669-713, 1997.

3. A. Dontchev, "Local analysis of a newton-type method based on partial linearization," Lectures in Appl. Math. 32, Amer. Math. Soc. Providence, RI, pp. 295-306, 1996.

4. — "Local convergence of the newton method for generalized equations," C. R. Acad. Sci. Paris Sér. I Math.r. I Math., vol. 322, no. 4, pp. 327-331, 1996.

5. A. Piétrus, "Generalized equations under mild differentiability conditions," Rev. R. Acad. Cienc. Exactas Fis. Nat., vol. 94, no. 1, pp. 15-18, 2000.

6. — - "Does newton's method for set-valued maps converge uniformly in mild differentiability context ?" Rev. Colombiana Mat., vol. 34, pp. 49-56, 2000.

7. M. Geoffroy, S. Hilout, and A. Piétrus, "Acceleration of convergence in dontchev's iterative method for solving variational inclusions," Serdica Math. J., vol. 29, no. 1, pp. 45-54, 2003.

8. — "Stability of a cubically convergent method for generalized equations," Set-valued Analysis, vol. 14, no. 1, pp. 41-54, 2006.

9. M. Geoffroy and A. Piétrus, "A superquadratic method for solving generalized equations in the holder case," Ricerce Mat., vol. 52, no. 2, pp. 231-240, 2003.

10. C. Jean-Alexis, "A cubic method without second order derivative for solving variational inclusions," $C . R$. Acad. Bulgare Sci., vol. 59, no. 12, pp. 1213-1218, 2006.

11. M. Geoffroy, C. Jean-Alexis, and A. Piétrus, "A hummel-seebeck type method for variational inclusions," Optimization, vol. 58, no. 4, pp. 389-399, 2009.

12. C. Cabuzel and A. Piétrus, "Solving variational inclusions by a method obtained using a multipoint iteration formula," Rev. Mat. Comput., vol. 22, no. 1, pp. 63-74, 2009.

13. J. Traub, Iterative methods for the solution of equations. New-York: Chelsea Publishing company, 1985.

14. M. Rashid, "Convergence analysis of extended hummel-seebeck type method for solving variational inclusions," Vietnam J. Math., vol. 44, no. 4, pp. 709-726, 2016. 
15. J. Aubin, "Lipschitz behavior of solutions to convex minimization problems," Math. Oper. Res, vol. 9, no. 1, pp. $87-111,1984$.

16. J. Aubin and H. Frankowska, Set-valued analysis. Boston: Birkhauser, 1990.

17. A. Dontchev, M. Quincampoix, and N. Zlateva, "Aubin criterion for metric regularity," J. Convex Anal., vol. 13 , no. 2 , pp. 281-297, 2006.

18. A. Dontchev and R. Rockafellar, "Characterizations of strong regularity for variational inequalities over polyhedral convex sets," SIAM J. Optim., vol. 6, no. 4, pp. 1087-1105, 1996.

19. _ _ " Regularity and conditioning of solutions mappings in variational analysis," Set-Valued Anal., vol. 12, no. 1-2, pp. 79-109, 2004.

20. - Implicit functions and solution mappings. Springer monographs in Mathematics, 2009.

21. B. Mordukhovich, "Complete characterization of openness metric regularity and lipschitzian properties of multifunctions," Trans. Amer. Math. Soc., vol. 340, no. 1, pp. 1-35, 1993.

22. - "Stability theory for parametric generalized equations and variational inequalities via nonsmooth analysis," Trans. Amer. Math. Soc., vol. 343, no. 2, pp. 609-657, 1994.

23. S. M. Robinson, "Strongly regular generalized equations," Math. Oper. Res., vol. 5, no. 1, pp. 43-62, 1980.

24. R. Rockafellar and J. Wets, Variational Analysis. Berlin: Springer-Verlag, 1998.

25. S. Adly and J. V. Outrata, "Qualitative stability of a class of non-monotone variational inclusions. application in electronic." Journal of Convex Analysis, vol. 20, no. 1, pp. 43-66, 2013.

26. J.-F. Bonnans, "Local analysis of newton-type methods for variational inequalities and nonlinear programming," Appl. Math. Optim., vol. 29, pp. 161-186, 1994.

27. S. Burnet, C. Jean-Alexis, and A. Piétrus, "An iterative method for semistable solutions," RACSAM, vol. 105, no. 1, pp. 133-138, 2011.

28. - - "A multipoint iterative method for semistable solutions," Appl. Math. E-notes, vol. 12, no. 44-52, 2012.

29. S. Burnet and A. Piétrus, "Local analysis of a cubically convergent method for variational inclusions," Appl. Mat., vol. 38, no. 2, pp. 183-191, 2011.

30. C. Cabuzel, A. Piétrus, and S. Burnet, "Local convergence of exact and inexact newton's method for subanalytic variational inclusions," Rev. Mat. Teor. Apl., vol. 22, no. 1, pp. 31-47, 2015.

31. F. J. Aragon, A. L. Dontchev, and M. H. Geoffroy, "Convergence of the proximal point method for metrically regular mappings," ESAIM Proc., vol. 17, pp. 1-8, 2007.

32. A. Dontchev and R. Rockafellar, "Newton's method for generalized equations: a sequential implicit function theorem," Math. Program., vol. 123, no. 1, pp. 139-159, 2010.

33. M. Gaydu and M. Geoffroy, "Tikhonov regularization of metrically regular inclusions," Positivity, vol. 13, no. 2, pp. 385-398, 2009.

34. M. Geoffroy, "An inertial proximal scheme for nonmonotone mappings," J. Math. Anal. Appl., vol. 350, no. 1, pp. 147-156, 2009.

35. M. Friedlander and M. Saunders, "A globally convergent linearly constrained lagrangian method for nonlinear optimization," SIAM J. Optim., vol. 15, pp. 863-897, 2005. 